OPEN ACCESS

Edited by:

Eric G. Cosio,

Pontifical Catholic University of Peru,

Peru

Reviewed by:

Georgios Liakopoulos,

Agricultural University of Athens,

Greece

Astrid Wingler,

University College Cork,

Ireland

*Correspondence:

Ling-Zeng Meng

lingzeng_meng@126.com

Wei-Chang Gong

gongweichang2008@163.com

Specialty section: This article was submitted to

Functional Plant Ecology,

a section of the journal

Frontiers in Plant Science

Received: 03 August 2019

Accepted: 21 January 2020

Published: 18 February 2020

Citation:

Gong $W-C$, Liu $Y-H$, Wang $C-M$, Chen $Y-Q$, Martin $K$ and Meng $L-Z$ (2020) Why Are There so Many Plant Species That Transiently Flush Young Leaves Red in the Tropics?

Front. Plant Sci. 11:83. doi: $10.3389 /$ fpls.2020.00083

\section{Why Are There so Many Plant Species That Transiently Flush Young Leaves Red in the Tropics?}

\author{
Wei-Chang Gong ${ }^{1 *}$, Yan-Hong Liu ${ }^{1}$, Chuan-Ming Wang ${ }^{1}$, Ya-Qing Chen ${ }^{1}$, Konrad Martin ${ }^{2}$ \\ and Ling-Zeng Meng ${ }^{1,2,3 *}$
}

${ }^{1}$ College of Life Science \& Technology, Honghe University, Mengzi, China, ${ }^{2}$ Institute of Agricultural Sciences in the Tropics, Hans-Ruthenberg-Institute, University of Hohenheim, Stuttgart, Germany, ${ }^{3}$ Xishuangbanna Tropical Botanical Garden, The Chinese Academy of Sciences, Mengla, China

Delayed greening of young leaves is a ubiquitous and visually striking phenomenon in the tropics. Here, we investigated the potential ecological functions of red coloration patterns in young leaves. To detect any protective function of the red coloration on the young leaves, leaf damage by insect herbivores was recorded in the field. To determine capacity for chemical defense, the concentrations of tannins and anthocyanins were measured in both young and mature leaves. To test the hypothesis that anthocyanins function as photoprotective molecules, chlorophyll content, maximum photochemical efficiency of $P S I I$ $\left(F_{v} / F_{m}\right)$, non-photochemical quenching $(N P Q)$, and effective quantum yield of $P S / I\left(\Phi_{P S I I}\right)$ were measured in the field. Phylogenetic relationships were analyzed to test the relationary significance of the occurrence of redness in young leaves. Compared to the coloration in non-red leaves, young red leaves had significant higher anthocyanins and tannins content and lower herbivore damages. Young, red leaves had the lowest $F_{v} / F_{m}$ values, which were significantly lower than those of non-red leaves. NPQ values in young red leaves were comparable to those of other groups. Although young red leaves had high $\Phi_{P S I I}$, these values were significantly lower than those of the other three groups. The results suggest that the red coloration of young leaves protects them from insect herbivory primary by chemical defense through high concentrations of tannins and anthocyanins. Additionally, low $F_{v} / F_{m}$ values in young red leaves indicate that anthocyanins might not be functioning as light attenuators to compensate for insufficient photo-protection mediated by NPQ. And finally, red coloration in young leaves is predominantly a result of adaptation to heavy herbivory stress but without significant intrinsic phylogenetic relationship of plant species.

Keywords: red coloration, young leaves, tropics, plant defense, anthocyanins, tannins

\section{INTRODUCTION}

Plant species are sensitive to changes in environmental stimuli and shift their physiological development to adapt to their specific surroundings (Niu et al., 2014). Environmental changes can alter the availability of resources and other conditions crucial to plant performance (Nicotra et al., 2010). Plant leaves are not only the most important photosynthetic organs but also the parts of 
the plant that can most sensitively respond to environmental changes (Nicotra et al., 2010; Niu et al., 2014). In the field, leaf size, shape, thickness, and folding behavior (Parkhurst and Loucks, 1972; Givnish, 1987; Turner, 1994; Smith et al., 1997; Beerling et al., 2001; Cornelissen et al., 2003; Huang et al., 2012), and even secondary metabolites or leaf coloration (Coley and Aide, 1989; Gould et al., 1995; Westoby, 1998; Nicotra et al., 2010; Chen and Huang, 2013; Niu et al., 2014; Tellez et al., 2016; Wang et al., 2016) can all be altered to respond to abiotic environmental changes (e.g., UV light) or biotic herbivory stresses.

In the tropics, certain plant species have evolved speciesspecific leaf development patterns, such as delayed greening (Kursar and Coley, 1992). Young leaves in tropical regions worldwide often flush red synchronously during expansion (Dominy et al., 2002; Manetas et al., 2003). Red flushing can be a widespread and visually striking phenomenon in the tropics, with between $20 \%-40 \%$ of the woody species showing red flushed young leaves at a single site (Opler et al., 1980). Coley and Barone (1996) reported that approximately one third of the plant species in tropical forests delayed greening of their young leaves until full expansion. Kursar and Coley (1992) found that $36 \%$ of tree species exhibited red coloration of young leaves at Barro Colorado Island (BCI), Panama. Furthermore, Chapman et al. (1999) reported that nearly $49 \%$ of forest trees in Kibale National Park, Uganda exhibited redness of young leaves at some point over the years. The delayed greening phenomenon in tropical rainforests of Southeast Asia included as much as $62 \%$ of the total studied plant species (Dominy, 2002).

Young red leaves generally contain high levels of anthocyanins, which are the primary contributors to the red coloration of plant leaves (Field et al., 2001; Trojak and Skowron, 2017; Cooney et al., 2018). Anthocyanins are produced not only in response to seasonal, spatial or developmental factors but can also be induced by a number of disparate abiotic (e.g., ultraviolet (UV) light) and biotic (e.g., insect herbivores, folivorous mammals, pathogens) stimuli (Hammerschmidt, 1977; Hipskind et al., 1996; Merzlyak and Chivkunova, 2000; Gould et al., 2002a; Trojak and Skowron, 2017). During leaf expansion, anthocyanins are frequently present in mesophyll and epidermal cells and then disappear from the tissues or decrease in concentration after full leaf expansion (Trojak and Skowron, 2017). In plants, anthocyanins function to screen against harmful reactive oxygen species (ROS), herbivore attacks, fungal attacks, and high light stress (Coley and Aide, 1989; Gould et al., 1995; Gould et al., 2002b; Tellez et al., 2016). However, anthocyanins do not serve as photosynthetic pigments (Trojak and Skowron, 2017). Since a primary function of plant leaves is photosynthesis, why do so many plant species not show an optimized pigment pool, (in terms of the absorption spectrum required for efficient light capture for photosynthesis) but instead accumulate high concentrations of anthocyanins and delay greening at the juvenile stage?

The ecological significance of delayed greening in young leaves has been disputed (Coley and Kursor, 1996). Coley and Aide (1989) found that leaf-cutter ants preferentially pick up leaves without anthocyanins. These authors then proposed the hypothesis that anthocyanins are associated with against invasions by leaf-attacking fungal pathogens. This view was shared by Tellez et al. (2016). Stone (1979) reported that the redness of young leaves may make them invisible to herbivores or may warn potential herbivores of the presence of toxic compounds. Other researchers have found that anthocyanins may function as light attenuators to compensate for insufficient photo-protection mediated by non-photochemical quenching (NPQ) (Demmig-Adams and Adams, 1992; Gould et al., 1995; Hughes et al., 2005; Zhu et al., 2018). However, these hypotheses of delayed greening in young leaves were put forward or verified based on studies considering only a few plant species, and whether these mechanisms have widespread adaptive significance for tropical plants is still unclear.

Plant-herbivore interactions constitute an important component of tropical rainforest biodiversity. Reciprocal selection has led to greater investment in defenses in tropical trees than in temperate plant species (Levin and York, 1978; Coley and Aide, 1989; Basset, 1994; Marquis and Braker, 1994). Although plant leaves are easily damaged by generalist or specialist herbivores (Cates, 1980; Coley and Aide, 1989; Broadway and Colvin, 1992; van Dam et al., 1995; Coley and Barone, 1996; Blüthgen and Metzner, 2007), young leaves, especially those with red phenotypes, can effectively decrease herbivore damage (Kursar and Coley, 1991; Karageorgou and Manetas, 2006). However, Chen and Huang (2013) reported that young red leaves have fewer mechanical physical defenses than do green leaves. Thus, the mechanism by which red coloration of young leaves helps them avoid harm from herbivores in the tropics is an important area of phenotypic study.

The tropics usually experience greater natural selection than temperate areas. The strong intensity of natural selection processes in tropical areas, which can be either continuous or intermittent processes consisting of exacerbations and remissions, makes phylogenetic relatedness of plant species decreased in tropical areas. Moreover, these ecological factors and their evolutionary relationships with plant species in tropical rainforests have resulted in an impressive variety of adaptations and interactions (Coley and Barone, 1996).

In the present study, we investigate the metabolism, photosynthetic activities and chemical defenses of leaves from 250 tropical plant species with either red or green young leaves. Our major aim is to investigate why there are so many plant species with transiently red young leaves in the tropics. Additionally, we attempt to highlight any ecological or evolutionary significance of delayed greening in young leaves of plants in the tropics. Our study involved systematic data collection in a tropical region of southwest China, through which we aim to answer the following questions:

1. Is red coloration in young leaves associated with the phylogenetic relationship of plant species?

2. Do young red leaves have better protection against herbivores than green leaves?

3. Does red coloration play a role in photosynthesis and photoprotection? 


\section{MATERIALS AND METHODS}

\section{Study Sites and Leaf Damage Investigation}

This work was carried out between January 2016 and March 2017 in Southern Yunnan, China, with most plant samples coming from Xishuangbanna Tropical Botanical Garden $\left(21^{\circ}\right.$ $41^{\prime} \mathrm{N}$ and $101^{\circ} 25^{\prime} \mathrm{E}, 570 \mathrm{~m}$ a.s.l.) and a small number of samples, used for field observation of natural leaf damage, from the botanic garden of Honghe University $\left(23^{\circ} 21^{\prime} \mathrm{N}\right.$ and $103^{\circ} 25^{\prime} \mathrm{E}$, $1331 \mathrm{~m}$ a.s.l).

To differentiate the effect that the hydrolysable tannin content in plants had on food plant preference in insect herbivores, an extensive field investigation in situ into the feeding patterns of insect herbivores feeding on leaves between early and late stages of maturation were carried out. Our field survey involved 83 redflushing woody species from 36 families (here called delayedgreening leaves, DGL) and 167 woody species without red young leaves (here called green leaves, GL) from 70 families. Prior to the experiment, three to five trees from each taxon were selected. Herbivore damage to young and mature leaves in each tree was assessed. For each plant, 10 branches from different parts of the tree were selected, and a total of approximately 200 young and mature leaves were assessed for herbivore damage. Irregularly shaped or incomplete leaves were recorded as damaged. The third and the fourth expanding leaves from the apex of each branch were treated as young leaves. The other leaves after the seventh from the apex of each branch were regarded as mature leaves.

\section{Anthocyanins and Chlorophyll Content}

We then determined the relative content of anthocyanins and the total chlorophyll content in both mature and young leaves, in order to investigate potential differences in anthocyanin and chlorophyll content between young and mature leaves. From the 250 plant species used in the field investigation into herbivory damage, we selected a total of 210 plant species (66 families, 150 genera), including DGL plant taxa (124 taxa, 43 families, 89 genera) and GL taxa (86 taxa, 42 families, 74 genera) to measure their concentrations of both anthocyanins and chlorophyll.

\section{Determination of Concentration of Anthocyanins}

The relative concentration of anthocyanins was determined following Christie et al. (1994) and Zhu et al. (2015) with minor modifications. Pulverized leaf blade tissue $(1.0 \mathrm{~g}$ fresh weight) was homogenized in $12 \mathrm{ml}$ of methanol containing $0.1 \mathrm{~N}$ HCL and maintained at $4^{\circ} \mathrm{C}$ for $4 \mathrm{~h}$. Three repetitions were performed for each taxon. Particulates were removed by centrifugation at $1000 \mathrm{r} / \mathrm{min}$ for $10 \mathrm{~min}$, and the absorption of the anthocyanin extracts was measured by a spectrophotometry (UV 5100B, Shanghai Metash Instrument Co., Ltd., Shanghai, China) at $530 \mathrm{~nm}$. The relative concentration of anthocyanins was then calculated as $10 \times \mathrm{A}_{530} \times \mathrm{B}$, where $\mathrm{A}$ is the measured absorption at $530 \mathrm{~nm}$, and $\mathrm{B}$ is the dilution ratio of the experimental supernatant.

\section{Chlorophyll Determination}

Chlorophyll was extracted by using $\mathrm{N}$, N-dimethylformamide, following Niu et al. (2014). The appropriate leaf area $\left(0.2 \mathrm{~cm}^{2}\right.$, fresh) of material for each species was punched from fresh leaves (avoiding major leaf veins where possible) through a circular leaf disc puncher $(\varnothing=5 \mathrm{~mm})$. Six $0.2 \mathrm{~cm}^{2}$ leaf disks were collected from six different leaves for each taxon, weighed fresh (to within $0.1 \mathrm{mg}$ ) were used and were then immersed in $\mathrm{N}, \mathrm{N}$ dimethylformamide for one night $(\sim 12 \mathrm{~h})$ in the dark. Three repetitions were performed for each taxon. The resulting solutions were then centrifuged at $1000 \mathrm{r} / \mathrm{min}$ for $10 \mathrm{~min}$, the supernatant was collected and the absorption values were measured using ultraviolet-visible spectrophotometry at 664.5 and $647 \mathrm{~nm}$. The concentration of chlorophyll was calculated as equivalents per fresh weight of the leaves following Inskeep and Bloom (1985) and Niu et al. (2014).

\section{Measurement of Total Tannin Content}

Tannic acid is regarded as a major component of the total phenolics within the leaf. Total phenolic content was determined using the Folin-Ciocalteau method, following Singleton and Rossi (1965) and Greer et al. (2014). Pulverized leaf blade tissue ( $0.5 \mathrm{~g}$ freeze-dried weight) was homogenized in $10 \mathrm{ml}$ of ethyl alcohol $(60 \%)$ and then heated in a water bath $\left(60^{\circ} \mathrm{C}\right)$ for $3 \mathrm{~h}$. Samples were weighed $( \pm 0.1 \mathrm{mg})$ to obtain their fresh weight (FW) before extraction. Three repetitions were completed for each taxon. Particulates were removed by centrifugation at $1000 \mathrm{r} / \mathrm{min}$ for $10 \mathrm{~min}$ and then filtered through a Buchner funnel. $1.0 \mathrm{ml}$ of the solution was then combined with $60 \mathrm{ml}$ of $\mathrm{dH}_{2} \mathrm{O}$ water, $5.0 \mathrm{ml}$ of FolinCiocalteau reagent and $15 \mathrm{ml}$ of sodium carbonate in a $100 \mathrm{ml}$ volumetric flask. A phenolic concentration standard curve was generated with tannic acid (concentrations from 0 to $50 \mathrm{mg} / \mathrm{L}$ ). To better fit the standard curve, leachates were diluted to $8 \times$ their original concentrations. Both standard and samples were analyzed in a spectrophotometer at $765 \mathrm{~nm}$. Total phenols are therefore reported as milligrams of tannic acid (gallotannin, Sigma) equivalents per gram fresh tissue (mg TAE/g FW).

\section{Chlorophyll a Fluorescence Activity}

Chlorophyll a fluorescence parameters of young and mature leaves were investigated using an Li-6400XT portable photosynthesis system (LiCOR Inc., USA) with an integrated leaf chamber fluorometer (LCF). For this experiment, of the total 250 plant species mentioned, 133 plant species were used, including 71 DGL species and 62 GL species. Ten leaves (five young and five mature leaves) from each taxon were used separately for the assessment of chlorophyll a fluorescence.

The maximum photochemical efficiency of PSII $\left(F_{v} / F_{m}\right)$, which can be used to quantify photo-inhibition (RedondoGómez et al., 2006), was then calculated following Figueroa et al. (1997) and Lima et al. (2018). On the following day (9:00-11:00), after light adaptation for half an hour, chlorophyll a fluorescence traits of the same leaves were estimated with the Li-6400XT portable photosynthesis system. Minimum fluorescence $\left(F_{0}\right)$ was measured under a continuous 
measuring light $\left(1200 \mu \mathrm{mol}\right.$ quanta $\left.\mathrm{m}^{-2} \mathrm{~s}^{-1}\right)$, and then $F_{m}$ was measured following a pulse of actinic light of $5000 \mu \mathrm{mol} \mathrm{m} \mathrm{m}^{-2}$. Maximum quantum yield of PSII was calculated as

$$
F_{v} / F_{m}=\left(F_{m}-F_{0}\right) / F_{m}
$$

After $30 \mathrm{~min}$ to allow for dark adaptation, the chlorophyll fluorescence parameters $F_{0}, F_{m}$, and $F_{s}$ of each of the young and mature leaves were measured. $F_{0}$ is the minimum chlorophyll a fluorescence after dark adaptation; $F_{m}$ is the maximal fluorescence level in the dark-adapted state, and $F_{s}$ is the steady-state fluorescence yield (Lima et al., 2018). The effective quantum yield of PSII $\left(\Phi_{P S I I}\right)$, which can be used for routine assays of plant heath performance and the quantification of environmental stress, was calculated following Redondo-Gómez et al. (2006) and Lima et al. (2018):

$$
\Phi_{\text {PSII }}=\left(F_{m^{\prime}}-F_{s}\right) / F_{m^{\prime}}
$$

Non-photochemical quenching $(N P Q)$, which is an important photo-protective process in plants, was also calculated as follows (Redondo-Gómez et al., 2006; Ware et al., 2015; Lima et al., 2018):

$$
N P Q=\left(F_{m}-F_{m^{\prime}}\right) F_{m^{\prime}}
$$

\section{Phylogenetic Relationships Between DGL and GL Taxa}

The family and genus names of all the studied species (210 species in total) in the APG III system were obtained using the $\mathrm{R}$ package plantlist (Zhang, 2018). The phylogenetic relationships between these species were examined using the online tool Phylomatic (Webb and Donoghue, 2005) (www.phylodiversity.net/ phylomatic/) based on the Angiosperm consensus tree from Davies et al. (2004). The results were visualized uisng the iTOL (itol.embl.de) online tool for the display and annotation of phylogenetic trees (Letunic and Bork, 2006; Letunic and Bork, 2016). A total of 204 species were shown in the tree, and six species (Balakata baccata, Lagerstroemia siamica, Lithocarpus microspermus, Nyssa sinensis, Parakmeria yunnanensis, Stixis suaveolens) were not because of no record in database. Branch lines and the background of the terminal nodes were colored, displaying DGL and GL plant species. Ten different color strings outside of the tree illustrated the family subtrees, including more than five species. The numerators and denominators of the fractions behind the family names give the number of DGL species and the total number of species studied in this family, respectively.

\section{Statistical Analyses}

For each measured parameter, the mean values for each species were used for further calculation and for presentation in figures. A list of all the plant species used in the measurement of photosynthesis physiology or determination of defense chemical content is supplied in the supporting information (Table S1). Nonparametric Kruskal-Wallis ANOVAs were conducted to test for differences in the percentage variation of leaf area damaged by insect herbivores between the young red and mature green leaves. GL plant species were compared in the same way. The effects of the color of the young leaf and species on the chlorophyll fluorescence parameter which shown the photosynthetic ability and potential protection mechanism, including $F_{\mathrm{v}} / F_{\mathrm{m}}$, effective $P S I I$ efficiency, and nonphotochemical dissipation (NPQ) were evaluated using twoway analysis of variance (ANOVA). Differences in anthocyanin, chlorophyll and tannin content between young and mature leaves were assessed using an $F$ test after all sample data had been tested for normality. A general Pearson linear regression and a Spearman's Rho non-parametric correlation were used to examine any potential relationships between the contents of anthocyanin and tannin in leaves, respectively. All of the statistical analyses were performed with the SPSS v.16.0 statistical package (SPSS, Chicago, IL). As well as the phylogenetic correlation analysis described above, we used two-tailed $U$-tests to compare the proportions of DGL and GL plant species in each family of the phylogenetic branch, to test the null hypothesis that of the color of the young leaves was not associated with their phylogenetic placement. The numbers present and absent in green species were set as the expected numbers. Figures were generated using SigmaPlot software (Version 14.0, Systat Software, San Jose, CA).

\section{RESULTS}

\section{Field Investigation of Leaf Damage}

Herbivore damage to young and mature leaves of plant species was investigated in the field (Figure 1). Young leaves of both DGL and GL species showed significantly lower herbivore damage proportions than did the mature leaves on the same plants $(P<0.001 ; P<0.001$, total $\mathrm{n}=250)$. The young GL leaves were more attacked by insect herbivores $(26.89 \pm 2.72 \%, \mathrm{n}=167)$ than young DGL leaves $(17.75 \pm 3.30 \%, \mathrm{n}=83)(P<0.01)$. Approximately 39\% $(n=83)$ of the green mature leaves from DGL plant species were damaged by herbivores, and 38\% ( $\mathrm{n}=$ 167) of the green mature leaves of GL plant species were damaged. There was no significant difference between DGL and GL groups $(P>0.05)$ (Figure 1).

\section{Leaf Chlorophyll, Anthocyanin and Tannin Characteristics}

We determined the levels of anthocyanins, tannin and chlorophyll characteristics in young and mature leaves (Figure 2). Both young leaves from DGL $(n=124)$ and from GL $(n=86)$ were investigated.

Anthocyanins in young and mature leaves were also evaluated, as shown in Figure 2A. Young leaves with red coloration had the greatest anthocyanin concentration, Around $6.45 \pm 0.66 \mathrm{~A}_{530 \mathrm{~nm}} / \mathrm{g} \mathrm{FW}$ anthocyanins were detected in young DGL leaves. This value was significantly higher than those from the other three groups $\left(F_{3,417}=38.78, P<0.001\right)$. In contrast, both young and mature GL leaves had relatively low levels of anthocyanins, ranging from $1.76 \pm 0.23$ to $2.14 \pm 0.38 \mathrm{~A}_{530 \mathrm{~nm}} / \mathrm{g}$ FW (Figure 2A). There were no significant differences between these three groups $(P>0.05)$. 


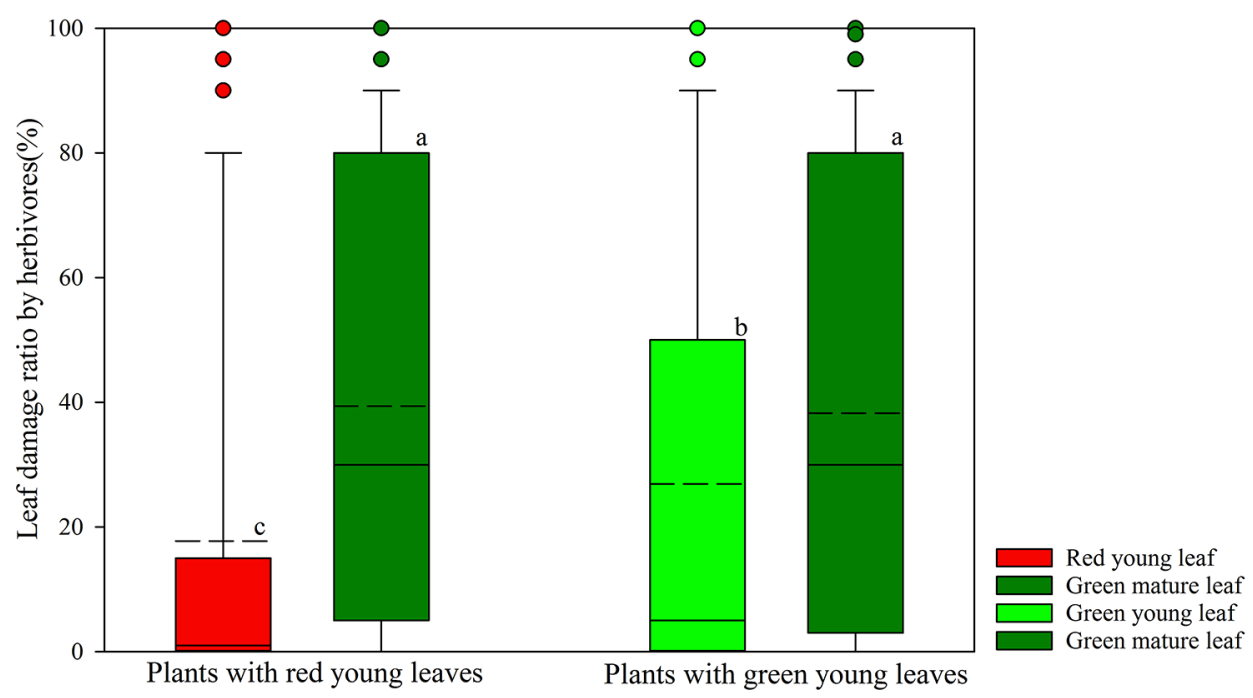

FIGURE 1 | Differences of leaf damage by herbivores in young and mature leaves in red- and green-young leaf taxa. Box and whisker plots illustrate the 5th, 25th, 50th (median), 75th, and 95th percentiles, and the means are given as a short dashed line. Different letters indicate significant differences $(P<0.05$; Nonparametric Kruskal-Wallis ANOVAs test). Black small circles represent outliers.

The tannin content of young and mature leaves is presented in Figure 2B, and shows the same trend as the leaf anthocyanin (Figure 2A). The highest tannin content was observed in young DGL leaves (13.81 mg TAE/g FW), which was significantly greater than values from the three non-red groups $\left(F_{3,417}=\right.$ 34.6, $P<0.001)$. The mature leaves from DGL taxa showed the second highest level of tannin (7.86 mg TAE/g FW) (Figure 2B). The leaf tannin concentrations in GL taxa were significantly lower than those in DGL taxa. No significant differences between young and mature leaves were found ( $t$-test, $P>0.05$ ).

The total chlorophyll content varied significantly between the four groups $(P<0.001$; Figure 2C). Mature leaves from all studied species had greater total chlorophyll content than did young leaves from the same species (Figure 2C). The total chlorophyll content was $0.14 \mathrm{mg} / \mathrm{g}$ FW in GL taxa. Moreover, mature leaves of DGL plant taxa contained more chlorophyll than young leaves from those two groups $(P<0.01)$. Additionally, the young DGL leaves had the lowest chlorophyll content, $0.09 \mathrm{mg} / \mathrm{g}$ (Figure 2C).

A significant correlation between anthocyanin and tannin contents of young DGL leaves was found $(r=0.20, P<0.05$, Pearson linear correlation; $r=0.30, P<0.01$, Spearman's $r s$ correlation; $n=96$, Figure 3A). However, this relationship was not observed in any of the other three groups $(P>0.05$, Figure 3).

\section{Chlorophyll Fluorescence Activity}

The chlorophyll a fluorescence of young and mature leaves was evaluated in the field (Figure 4). Young leaves from DGL ( $\mathrm{n}=$ $71)$ and GL $(n=62)$ species were used. Both young and mature GL leaves had high $F_{v} / F_{m}$ values, ranging from 0.74 to 0.79

(Figure 4A). Young DGL leaves had the lowest $F_{v} / F_{m}$ values,
0.74 , which were significantly lower than the values of the other three non-red leaf groups $(P<0.001)$. There were significant differences $(P<0.05)$ between young and mature leaves in GL leaf taxa.

The differences in $\Phi_{P S I}$, the quantum efficiency of PSII photochemistry, between young and mature leaves was assessed (Figure 4B). All studied taxa showed relatively high $\Phi_{P S I I}$ values, ranging from 0.70 to 0.78 . Young DGL leaves exhibited the lowest $\Phi_{P S I I}$ values, which were significantly different from the other three non-red groups $(P<0.001)$. A significant difference among GL leaf taxa was found $(P<0.05)$.

Differences in the $N P Q$ values between young and mature leaves were evaluated, ranging from 0.48 to 0.59 (Figure 4C). There were significant differences among the four groups $(P<$ $0.05)$. In red-young leaf taxa, $N P Q$ values in young red leaves were significant greater than in their mature leaves $(P<0.05)$. However, there were no significant differences between red- and green-young leaves $(P>0.05)$ (Figure 4C).

\section{Phylogenetic Reconstruction}

The phylogenetic relationships between the 204 studied species were reconstructed (Figure 5). The occurrence of redness in young leaves was not closely associated with their phylogenetic relationships. Plants with young leaves that flush red can be allied closely to other DGL taxa, or can belong to genera with many taxa with different coloration. However, the occurrence of redness in young leaves is a universal phenomenon. For example, 8 out of 14 studied species in the Euphorbiaceae showed young red leaves, 16 out of 23 in the Fabaceae, 11 out of 20 in the Moraceae and 6 out of 7 in the Myrtaceae. Additionally, all studied taxa from the Dipterocarpaceae, Fagaceae, Lythraceae, Myrtaceae, Rosaceae, and Salicaceae 

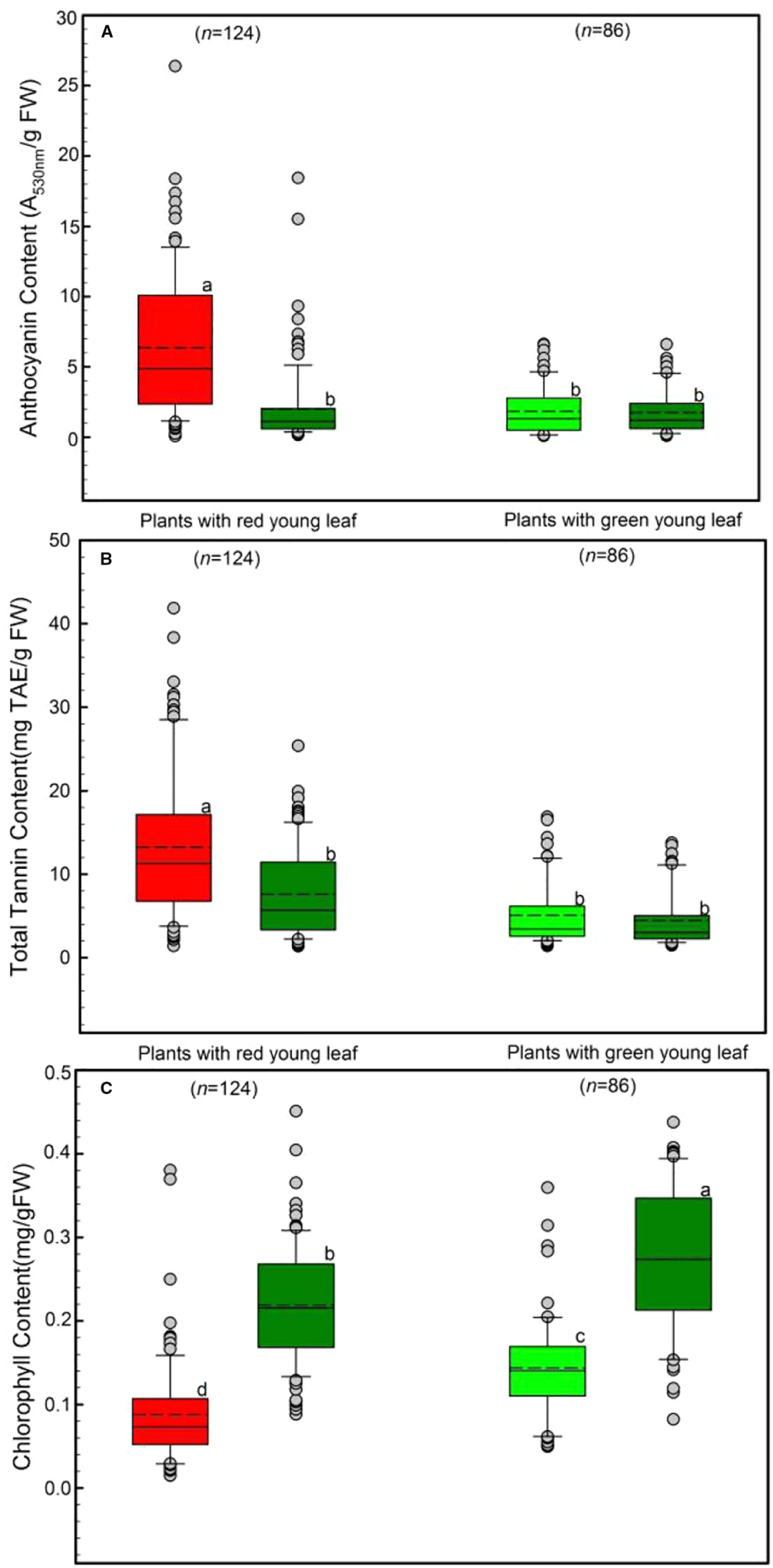

Plants with red young leaf

Plants with green young leaf

FIGURE 2 | Differences in leaf anthocyanin (A), tannin (B), and chlorophyll (C) concentrations of young and mature leaves in red- and green-young leaf taxa. Box and whisker plots illustrate the 5th, 25th, 50th (median), 75th, and 95th percentiles, and the means are given as a short dashed line. Different letters indicate significant differences $(P<0.05 ; F$-test). Grey small circle represent outliers. 

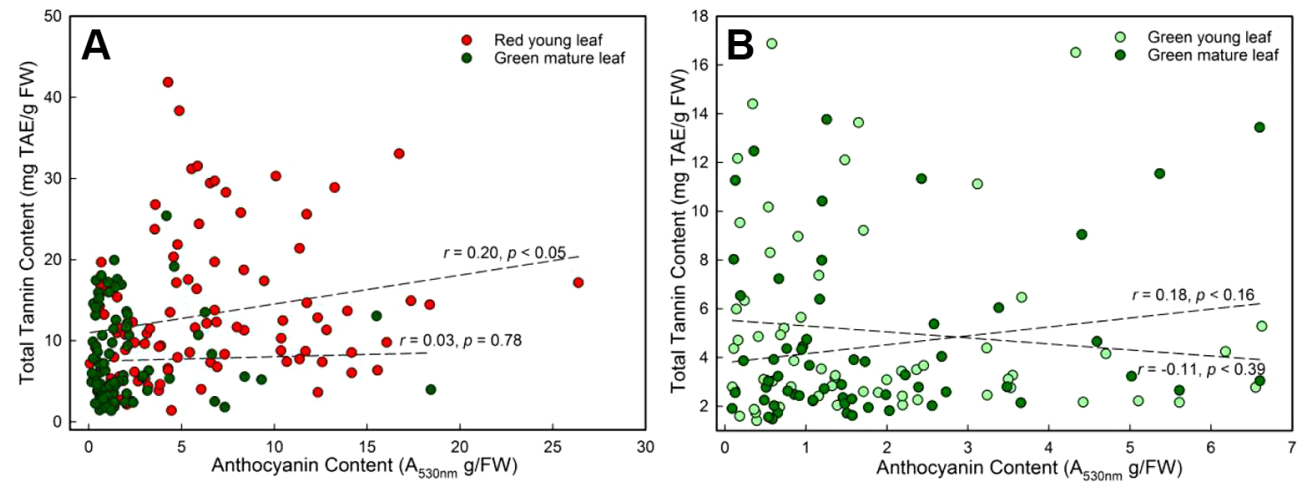

FIGURE 3 | The general Pearson linear correlation between leaf anthocyanin and tannin contents of young and mature leaves in red- and green-young leaf taxa. (A) red-young leaf taxa $(n=96) ;(B)$ green-young leaf taxa $(n=61)$.

exhibited red-colored young leaves. Conversely, we also found that all studied taxa in the Achariaceae and Piperaceae have green young leaves.

\section{DISCUSSION}

The anthocyanin pigments and the tannins, which are defense chemicals, are known to share the same biosynthetic pathway, the phenylpropanoid pathway (Ishikura et al., 1984). It is therefore not surprising that both have a relatively higher accumulation within red young leaves in the present research. Recent literature suggests that high levels of hydrolysable tannins [mainly galloylglucoses/ellagitannins (GGs/ETs)] in the young leaves of plants can inhibit POD mediated anthocyanin degradation and can increase stability and color intensity of anthocyanins by copigmentation activity (Luo et al., 2019). Previous studies have also reported that the delayed greening strategy is widespread in the tropics (Coley and Kursor, 1996; Dominy et al., 2002), and that young tropical leaves with visually striking red coloration have a greater increase in chemical defenses compared with mature leaves than do their temperate counterparts (Coley and Aide, 1989). The ecological function of anthocyanins is hotly debated. Our field observations show that young leaves from DGL plants can more efficiently escape insect herbivory with low leaf damage $(17.75 \pm 3.30 \%)$ compared to young GL leaves $(26.89 \pm 2.72 \%$, Figure 1). We were also able to confirm that young DGL leaves are better able to defend against insect herbivory than are mature leaves. We predict that a higher concentration of anthocyanins and tannins also may plays a role in screening against harmful UV radiation, and can enhance chemical defenses separately based on the generalization of functional ecological specificity.

Theoretically, the low toughness and high nutritive value of young leaves may mean that they are preferred by grazers. Chen and Huang (2013) reported that young red leaves have fewer mechanical defenses than green leaves. Moreover, some studies showed that insect herbivores caused higher leaf area damage to young leaves (Coley and Aide, 1989; Coley and Barone, 1996). These results do not contradict our findings from the Asiatic tropics. Generalist herbivores prefer mature leaves, but they show lower performance than specialist herbivores with specific secondary metabolites (Blüthgen and Metzner, 2007). Therefore, the young DGL leaves may selectively protect themselves through chemical defenses because they have not got physical defense mechanisms.

In the present study, DGL plant taxa produced high amounts of tannins and anthocyanins in those leaves, $13.81 \mathrm{mg} \mathrm{TAE} / \mathrm{g}$ FW, and $44 \mathrm{~A}_{530 \mathrm{~nm}} / \mathrm{g}$ FW, respectively (Figure 2). Tannins have been widely used as an index of plant chemical defense, as they considerably lower the palatability of leaf tissue (Lowman and Box, 1983). They can defend leaves against insect herbivores by deterrence or toxicity. At the same time, tannin-rich leaves contain high concentrations of anthocyanins because these components share the initial steps of the same synthetic pathway (Winkel-Shirley, 2002). Anthocyanins not only contribute to the redness of young leaves but also can effectively scavenge super-oxide and hydrogen peroxide generated by insect-mediated or mechanical damage through the browning reaction (Gould et al., 2002a).

In the tropical wild, plants face not only selection pressure from herbivory, but also abiotic environmental stresses, such as high light exposure, especially in the tropical rainforest zone. Excessive light can cause photo-inhibition, accompanied by damage to the photosynthetic apparatus and a decrease in the photosynthetic rate and can even cause photo-oxidation (Demmig-Adams and Adams, 1992).

A typical plant taxa has an $F_{v} / F_{m}$ that ranges from 0.75 to 0.85 (Björkman and Demmig, 1987; Hogewoning et al., 2012). In this study, young DGL leaves had the lowest $F_{v} / F_{m}$ ratio $(0.70)$ and all non-red leaves had relatively high $F_{v} / F_{m}$ values $(0.77 \sim 0.79)$ (Figure 3A). Red leaves exhibited strikingly lower $F_{v} / F_{m}$ ratios than did nonred leaves (Figure 3A). Thus, our results suggest the occurrence of photo-inhibition to some extent in young DGL leaves.

Plants can dissipate excessive light energy absorbed by lightharvesting complexes through non-photochemical quenching 

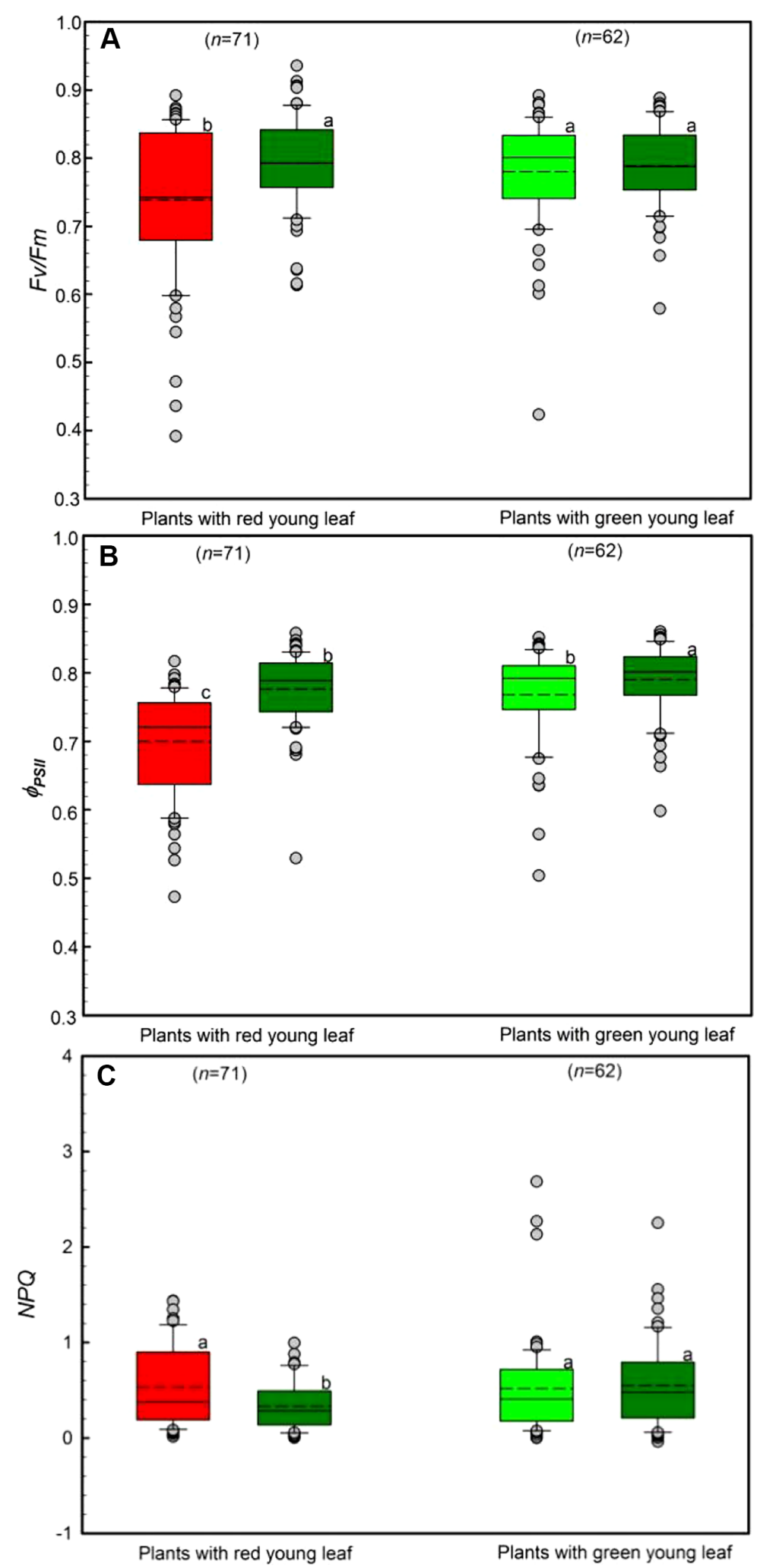

FIGURE 4 | Chlorophyll a fluorescence parameters including $F_{V} / F_{m}(\mathbf{A}), \Phi_{P S I /}(\mathbf{B})$ and $N P Q(\mathbf{C})$ of young and mature leaves in red- and green-young leaf taxa. Box and whisker plots illustrate the 5th, 25th, 50th (median), 75th, and 95th percentiles, and the means are given as a short dashed line. Different letters indicate significant differences $(P<0.05$; two way ANOVAs test). Grey small circle represent outliers. 


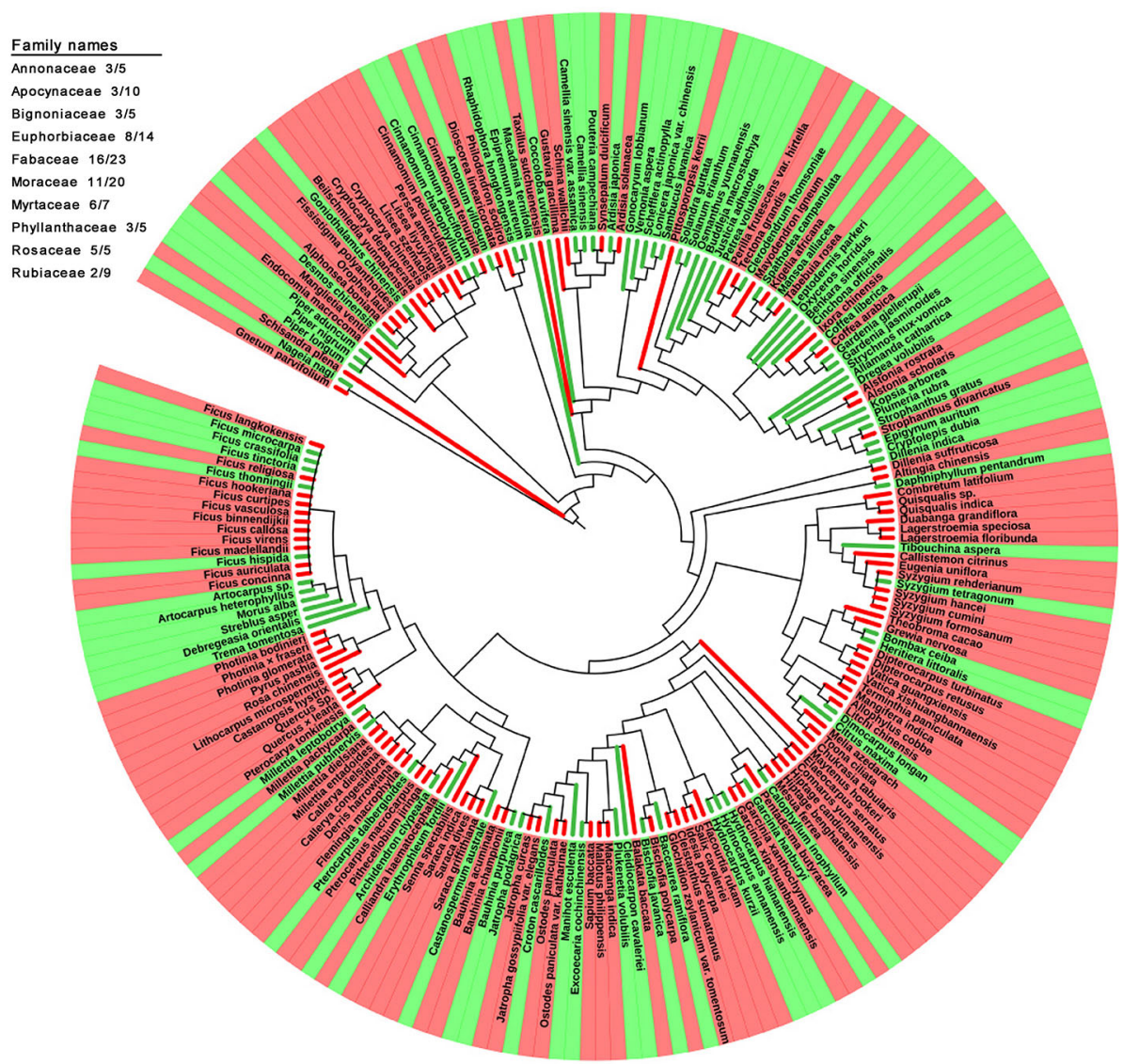

FIGURE 5 | Phylogenetic development tree map of 205 studied plant species with red and green young leaves.

$(N P Q)$, which is based on the xanthophyll cycle (Müller-Moulé et al., 2002; Tietz et al., 2017). NPQ as measured in this study does not represent the total capacity for non-photochemical quenching of the samples, but it is rather a measure of the instantaneous level of this process depending on the light incident on the sample surface, light absorptivity, energy pressure on the photosystems and photo-protective potential (i.e., xanthophyll pool size). The $\Phi_{P S I I}$ calculated was also an instantaneous measurement of the operational efficiency of the PSII photochemistry, and as such has little to do with the potential capacity for photo protection. In this study, young DGL leaves exhibited significantly greater $N P Q$ levels than mature green leaves from DGL plants, but displayed no significant differences from GL plant species (Figure 4C). This suggests that $N P Q$ could provide photo protection for redcolored young leaves to some extent. The young DGL leaves exhibited significantly lower $\Phi_{\text {PSII }}$ efficiency (0.74) than did GL leaves (Figure 4B). This result was closely associated with lower efficiency of light energy utilization, and suggests that the photosynthetic apparatus of young DGL leaves was not damaged by normal photosynthesis and that red leaves can most probably dissipate excess light energy through other mechanisms.
In addition to their role in $N P Q$, anthocyanins may also be able to function as ideal photo-protective agents because of their spectral absorption characteristics and anti-oxidative properties (Hughes et al., 2005; Zhu et al., 2018) although this is still in dispute. NPQ and anthocyanins are likely to be two important tools that can provide photo-protective function in plant leaves through different mechanisms. Anthocyanins-mediated photo-protection always occurs at species-specific developmental stages (Zhu et al., 2018). Combined with the high anthocyanin concentrations of young red leaves, anthocyanins may function as light attenuators and ameliorate the effects of excess light energy. However, the lowest $F_{v} / F_{m}$ values observed at young DGL leaves in this study showed that photo-inhibition to some extent occurred. Thus, the high concentrations of anthocyanin in young DGL leaves might not have compensated for the insufficient photo-protection mediated by $N P Q$ in these leaves but may enhance the fitness of young plants in tropical rainforests against herbivory damage.

\section{CONCLUSION}

In this interspecific, ecophysiological study, the occurrence of transient reddening of juvenile leaves in the tropics was coupled 
with increased levels of both anthocyanins and tannins. These two classes of compound were found to co-existed in transiently red young leaves and may have an intrinsic quantitative correlation mediated through POD enzyme activities.

Young leaves that flush red in tropical rainforest plants enhance plant defense and increase plant fitness. Moreover, the occurrence of red coloration in young leaves has arisen many times independently and is present in a variety of unrelated families (Figure 5). This indicates that red coloration in young leaves is predominantly a result of adaptation to special tropical environmental conditions but without significant intrinsic phylogenetic relationship of plant species. Our findings suggest that the red coloration of young leaves, which contain high concentrations of tannin and anthocyanin, mainly functions as an anti-herbivore defense strategy with chemical components. Furthermore, anthocyanins, which are principally responsible for the redness of young leaves, might not function as light attenuators to compensate for insufficient photo-protection mediated by NPQ because of the lowest $F_{v} / F_{m}$ values, but may enhance the fitness of young plants in tropical rainforests to defense herbivory damage.

\section{DATA AVAILABILITY STATEMENT}

All datasets for this study are included in the article/ Supplementary Material.

\section{AUTHOR CONTRIBUTIONS}

$\mathrm{W}-\mathrm{CG}$ and L-ZM planned and designed the research. W-CG, Y-HL, C-MW, Y-QC, and L-ZM collected the data. W-CG and L-ZM contributed to data compilation. W-CG analyzed the data

\section{REFERENCES}

Basset, Y. (1994). Palatability of tree foliage to chewing insects: a comparison between a temperate and a tropical site. Acta Oecologica 15, 181-191.

Beerling, D. J., Osborne, C. P., and Chaloner, W. G. (2001). Evolution of leaf-form in land plants linked to atmospheric $\mathrm{CO}_{2}$ decline in the Late Palaeozoic era. Nature 410, 352-354. doi: 10.1038/35066546

Björkman, O., and Demmig, B. (1987). Photon yield of $\mathrm{O}_{2}$ evolution and chlorophyll fluorescence characteristics at $77 \mathrm{~K}$ among vascular plants of diverse origins. Planta 170, 489-504. doi: 10.1007/BF00402983

Blüthgen, N., and Metzner, A. (2007). Contrasting leaf age preferences of specialist and generalist stick insects (Phasmida). Oikos 116, 1853-1862. doi: 10.1111/ j.0030-1299.2007.16037.x

Broadway, R. M., and Colvin, A. A. (1992). Influence of cabbage proteinase inhibitors in situ on the growth of larval Trichoplusia ni and Pieris rapae. J. Chem. Ecol. 18, 1009-1024. doi: 10.1007/BF00980059

Cates, R. G. (1980). Feeding patterns of monophagous, oligophagous, and polyphagous insect herbivores: The effect of resource abundance and plant chemistry. Oecologia 46, 22-31. doi: 10.1007/BF00346961

Chapman, C. A., Wrangham, R. W., Chapman, L. J., Kennard, D. K., and Zanne, A. E. (1999). Fruit and flower phenology at two sites in Kibale National Park, Uganda. J. Trop. Ecol. 15, 189-211. doi: 10.1017/S0266467499000759

Chen, Y.-Z., and Huang, S.-Q. (2013). Red young leaves have less mechanical defence than green young leaves. Oikos 122, 1035-1041. doi: 10.1111/j.16000706.2012.20852.x with support from L-ZM and C-M-W. W-CG, KM, and L-ZM wrote the manuscript with contributions from C-MW. All authors gave final approval for publication.

\section{ACKNOWLEDGMENTS}

We thank the two reviewers of this article for their valuable suggestions and advice. Chao-Hu Peng, Shan Liao, and YaCheng Wang from Honghe University helped in the field with the chlorophyll a fluorescence activity measurements at Xishuangbanna Tropical Botanical Garden (XTBG), The Chinese Academy of Sciences. Prof. Jiao-Lin Zhang in XTBG also provided suggestions, and some measurement data. This study was supported by funds from National Natural Science Foundation of China (grant no. NSFC-31200322; 31760171; 31660111), Honghe University (grant nos. XJ14Z01, XJ15B17, and XJ16B05), and the CAS 135 program (no. 2017XTBG-T01). The work of the fifth author (KM) was supported by the project SURUMER (Sustainable Rubber Cultivation in the Mekong Region), funded by the German Federal Ministry of Education and Research (BMBF) program "Sustainable Land Management”.

\section{SUPPLEMENTARY MATERIAL}

The Supplementary Material for this article can be found online at: https://www.frontiersin.org/articles/10.3389/fpls.2020.00083/ full\#supplementary-material

SUPPLEMENTARY TABLE 1 | Name list of plant species used in analysis of photosynthesis physiology and defense chemical content.

Christie, P. J., Alfenito, M. R., and Walbot, V. (1994). Impact of low-temperature stress on general phenylpropanoid and anthocyanin pathways: enhancement of transcript abundance and anthocyanin pigmentation in maize seedlings. Planta 194, 541-549. doi: 10.1007/BF00714468

Coley, P. D., and Aide, T. M. (1989). Red coloration of tropical young leaves: a possible antifungal defence? J. Trop. Ecol. 5, 293-300. doi: 10.1017/S0266467400003667

Coley, P. D., and Barone, J. A. (1996). Herbivory and plant defenses in tropical forests. Annu. Rev. Ecol. Evolution Syst. 27, 305-335. doi: 10.1146/annurev. ecolsys.27.1.305

Coley, P. D., and Kursor, T. A. (1996). "Anti-herbivore defenses of young tropical leaves: physiological constraints and ecological trade-offs," in Tropical Forest Plant Ecophysiology. Eds. S. S. Mulkey, R. L. Chazdon and A. P. Smith (Boston, MA: Springer US), 27, 305-336.

Cooney, L. J., Logan, B. A., Walsh, M. J. L., Nnatubeugo, N. B., Reblin, J. S., and Gould, K. S. (2018). Photoprotection from anthocyanins and thermal energy dissipation in senescing red and green Sambucus canadensis peduncles. Environ. Exp. Bot. 148, 27-34. doi: 10.1016/j.envexpbot.2017.12.019

Cornelissen, J. H. C., Lavorel, S., Garnier, E., Díaz, S., Buchmann, N., Gurvich, D. E., et al. (2003). A handbook of protocols for standardised and easy measurement of plant functional traits worldwide. Aust. J. Bot. 51, 335-380. doi: 10.1071/BT02124

Davies, T. J., Barraclough, T. G., Chase, M. W., Soltis, P. S., Soltis, D. E., and Savolainen, V. (2004). Darwin's abominable mystery: insights from a supertree of the angiosperms. Proc. Natl. Acad. Sci. 101, 1904-1909. doi: 10.1073/pnas. 0308127100 
Demmig-Adams, B., and Adams, W. W.III (1992). Photoprotection and other responses of plants to high light stress. Annu. Rev. Plant Physiol. Plant Mol. Biol. 43, 599-626. doi: 10.1146/annurev.pp.43.060192.003123

Dominy, N. J., Lucas, P. W., Ramsden, L. W., Riba-Hernandez, P., Stoner, K. E., and Turner, I. M. (2002). Why are young leaves red? Oikos 98, 163-176. doi: 10.1034/j.1600-0706.2002.980117.x

Dominy, N. J. (2002). Incidence of red leaves in the rainforest of Kibale National Park, Uganda: shade-tolerators and light-demanders compared. Afr. J. Ecol. 40, 94-96. doi: 10.1046/j.0141-6707.2001.00330.x

Feild, T. S., Lee, D. W., and Holbrook, N. M. (2001). Why leaves turn red in autumn. the role of anthocyanins in senescing leaves of red-osier dogwood. Plant Physiol. 127, 566-574. doi: 10.1104/pp.010063

Figueroa, M. E., Fernández-Baco, L., Luque, T., and Davy, A. J. (1997). Chlorophyll fluorescence, stress and survival in populations of Mediterranean grassland species. J. Vegetation Sci. 8, 881-888. doi: 10.2307/ 3237033

Givnish, T. J. (1987). Comparative studies of leaf form: assessing the relative roles of selective pressures and phylogenetic constraints. New Phytol. 106, 131-160. doi: 10.1111/j.1469-8137.1987.tb04687.x

Gould, K. S., Kuhn, D. N., Lee, D. W., and Oberbauer, S. F. (1995). Why leaves are sometimes red. Nature 378, 241-242. doi: 10.1038/378241b0

Gould, K. S., McKelvie, J., and Markham, K. R. (2002a). Do anthocyanins function as antioxidants in leaves? Imaging of $\mathrm{H}_{2} \mathrm{O}_{2}$ in red and green leaves after mechanical injury. Plant Cell Environ. 25, 1261-1269. doi: 10.1046/j.13653040.2002.00905.x

Gould, K. S., Neill, S. O., and Vogelmann, T. C. (2002b). A unified explanation for anthocyanins in leaves? Adv. Bot. Res. 37, 167-192. doi: 10.1016/S0065-2296 (02)37049-6

Greer, M. J., Wilson, G. W. T., Hickman, K. R., and Wilson, S. M. (2014). Experimental evidence that invasive grasses use allelopathic biochemicals as a potential mechanism for invasion: chemical warfare in nature. Plant Soil 385, 165-179. doi: 10.1007/s11104-014-2209-3

Hammerschmidt, R. (1977). Resistance of maize to anthracnose: changes in host phenols and pigments. Phytopathology 67, 251-258. doi: 10.1094/Phyto-67-251

Hipskind, J., Wood, K., and Nicholson, R. L. (1996). Localized stimulation of anthocyanin accumulation and delineation of pathogen ingress in maize genetically resistant toBipolaris maydisrace O. Physiol. Mol. Plant Pathol. 49, 247-256.

Hogewoning, S. W., Wientjes, E., Douwstra, P., Trouwborst, G., van Ieperen, W., Croce, R., et al. (2012). Photosynthetic quantum yield dynamics: from photosystems to leaves. Plant Cell 24, 1921-1935.

Huang, W., Zhang, S.-B., and Cao, K.-F. (2012). Evidence for leaf fold to remedy the deficiency of physiological photoprotection for photosystem II. Photosynth. Res. 110, 185-191.

Hughes, N. M., Neufeld, H. S., and Burkey, K. O. (2005). Functional role of anthocyanins in high-light winter leaves of the evergreen herb Galax urceolata. New Phytol. 168, 575-587.

Inskeep, W. P., and Bloom, P. R. (1985). Extinction coefficients of chlorophyll a and b in N,N-Dimethylformamide and 80\% acetone. Plant Physiol. 77, 483-485.

Ishikura, N., Hayashida, S., and Tazaki, K. (1984). Biosynthesis of gallic and ellagic acids with 14C-labeled compounds in Acer and Rhus leaves. Bot. Magazine 97 (3), 355-367. doi: 10.1007/BF02488668

Karageorgou, P., and Manetas, Y. (2006). The importance of being red when young: anthocyanins and the protection of young leaves of Quercus coccifera from insect herbivory and excess light. Tree Physiol. 26, 613-621. doi: 10.1093/ treephys/26.5.613

Kursar, T. A., and Coley, P. D. (1991). Nitrogen content and expansion rate of young leaves of rain forest species: implications for herbivory. Biotropica 23, 141-150. doi: 10.2307/2388299

Kursar, T. A., and Coley, P. D. (1992). Delayed greening in tropical leaves: an antiherbivore defense? Biotropica 24, 256-262. doi: 10.2307/2388520

Letunic, I., and Bork, P. (2006). Interactive Tree of Life (iTOL): an online tool for phylogenetic tree display and annotation. Bioinformatics 23, 127-128. doi: 10.1093/bioinformatics/btl529

Letunic, I., and Bork, P. (2016). Interactive tree of life (iTOL) v3: an online tool for the display and annotation of phylogenetic and other trees. Nucleic Acids Res. 44, W242-W245. doi: 10.1093/nar/gkw290
Levin, D. A., and York, B. M. (1978). The toxicity of plant alkaloids: an Ecogeographic perspective. Biochem. Syst. Ecol. 6, 61-76. doi: 10.1016/03051978(78)90026-1

Lima, C. S., Ferreira-Silva, S. L., Carvalho, F. E. L., Lima Neto, M. C., Aragão, R. M., Silva, E. N., et al. (2018). Antioxidant protection and PSII regulation mitigate photo-oxidative stress induced by drought followed by high light in cashew plants. Environ. Exp. Bot. 149, 59-69. doi: 10.1016/ j.envexpbot.2018.02.001

Lowman, M. D., and Box, J. D. (1983). Variation in leaf toughness and phenolic content among five species of Australian rain forest trees. Aust. J. Ecol. 8, 1725. doi: 10.1111/j.1442-9993.1983.tb01515.x

Luo, H., Li, W., Zhang, X., Deng, S.-F., Xu, Q.-C., Hou, T., et al. (2019). In planta high levels of hydrolysable tannins inhibit peroxidase mediated anthocyanin degradation and maintain abaxially red leaves of Excoecaria cochinchinensis. BMC Plant Biol. 19, 315. doi: 10.1186/s12870-019-1903-y

Müller-Moulé, P., Conklin, P. L., and Niyogi, K. K. (2002). Ascorbate deficiency can limit violaxanthin de-epoxidase activity in vivo. Plant Physiol. 128, $970-$ 977. doi: $10.1104 /$ pp.010924

Manetas, Y., Petropoulou, Y., K. Psaras, G., and Drinia, A. (2003). Exposed red (anthocyanic) leaves of Quercus coccifera display shade characteristics. Funct. Plant Biol. 30, 265-270. doi: 10.1071/FP02226

Marquis, R., and Braker, E. (1994). Plant-herbivore interactions: diversity, specificity, and impact. La Selva: Ecol. Natural History A Neotropical Rain For. Eds. McDade, L. A., Bawa, K. S., Hespenheide, H. A., and Hartshorn, G. S. (Chicago/London: University of Chicago Press), 261-281.

Merzlyak, M. N., and Chivkunova, O. B. (2000). Light-stress-induced pigment changes and evidence for anthocyanin photoprotection in apples. J. Photochem. Photobiol. B: Biol. 55, 155-163. doi: 10.1016/S1011-1344(00)00042-7

Nicotra, A. B., Atkin, O. K., Bonser, S. P., Davidson, A. M., Finnegan, E. J., Mathesius, U., et al. (2010). Plant phenotypic plasticity in a changing climate. Trends Plant Sci. 15, 684-692. doi: 10.1016/j.tplants.2010.09.008

Niu, Y., Chen, G., Peng, D.-L., Song, B., Yang, Y., Li, Z.-M., et al. (2014). Grey leaves in an alpine plant: a cryptic colouration to avoid attack? New Phytol. 203, 953-963. doi: 10.1111/nph.12834

Opler, P. A., Frankie, G. W., and Baker, H. G. (1980). Comparative phenological studies of treelet and shrub species in tropical wet and dry forests in the lowlands of Costa Rica. J. Ecol. 68, 167-188. doi: 10.2307/2259250

Parkhurst, D. F., and Loucks, O. L. (1972). Optimal leaf size in relation to environment. J. Ecol. 60, 505-537. doi: 10.2307/2258359

Redondo-Gómez, S., Wharmby, C., Castillo, J. M., Mateos-Naranjo, E., Luque, C. J., De Cires, A., et al. (2006). Growth and photosynthetic responses to salinity in an extreme halophyte, Sarcocornia fruticosa. Physiologia Plantarum 128, 116-124. doi: 10.1111/j.1399-3054.2006.00719.x

Singleton, V. L., and Rossi, J. A. (1965). Colorimetry of total phenolics with phosphomolybdic-phosphotungstic acid reagents. Am. J. Enol. Viticult. 16, 144-158.

Smith, W. K., Vogelmann, T. C., DeLucia, E. H., Bell, D. T., and Shepherd, K. A. (1997). Leaf form and photosynthesis. BioScience 47, 785-793. doi: 10.2307/ 1313100

Stone, B. C. (1979). Protective coloration of young leaves in certain Malaysian palms. Biotropica 11, 126-126. doi: 10.2307/2387788

Tellez, P., Rojas, E., and Van Bael, S. (2016). Red coloration in young tropical leaves associated with reduced fungal pathogen damage. Biotropica 48, 150153. doi: $10.1111 /$ btp. 12303

Tietz, S., Hall, C. C., Cruz, J. A., and Kramer, D. M. (2017). NPQ(T): a chlorophyll fluorescence parameter for rapid estimation and imaging of nonphotochemical quenching of excitons in photosystem-II-associated antenna complexes. Plant Cell Environ. 40, 1243-1255. doi: 10.1111/pce.12924

Trojak, M., and Skowron, E. (2017). Role of anthocyanins in high-light stress response. World Sci. News 81, 150-168.

Turner, I. M. (1994). A quantitative analysis of leaf form in woody plants from the world's major broadleaved forest types. J. Biogeogr. 21, 413-419. doi: 10.2307/ 2845759

van Dam, N. M., Vuister, L. W. M., Bergshoeff, C., de Vos, H., and van Der Meijden, E. (1995). The "Raison D'être" of pyrrolizidine alkaloids in Cynoglossum officinale: deterrent effects against generalist herbivores. J. Chem. Ecol. 21, 507-523. doi: 10.1007/BF02033698 
Wang, Y., Shao, L., Wang, J., Ren, H., Liu, H., M. Zhang, Q., et al. (2016). Comparison of morphological and physiological characteristics in two phenotypes of a rare and endangered plant, Begonia fimbristipula Hance. Photosynthetica 54, 381-389. doi: 10.1007/s11099-016-0199-5

Ware, M. A., Belgio, E., and Ruban, A. V. (2015). Photoprotective capacity of nonphotochemical quenching in plants acclimated to different light intensities. Photosynth. Res. 126, 261-274. doi: 10.1007/s11120-015-0102-4

Webb, C. O., and Donoghue, M. J. (2005). Phylomatic: tree assembly for applied phylogenetics. Mol. Ecol. Notes 5, 181-183. doi: 10.1111/j.14718286.2004.00829.x

Westoby, M. (1998). A leaf-height-seed (LHS) plant ecology strategy scheme. Plant Soil 199, 213-227. doi: 10.1023/A:1004327224729

Winkel-Shirley, B. (2002). Biosynthesis of flavonoids and effects of stress. Curr. Opin. Plant Biol. 5, 218-223. doi: 10.1016/S1369-5266(02)00256-X

Zhang, J.-L. (2018). plantlist: looking up the status of plant scientific names based on the plant list database. R package version 0.5.3. https://github.com/helixcn/plantlist/.

Zhu, Q.-L., Sui, S.-Z., Lei, X.-H., Yang, Z.-F., Lu, K., Liu, G.-D., et al. (2015). Ectopic expression of the Coleus R2R3 MYB-Type Proanthocyanidin regulator gene ssMYB3 alters the flower color in transgenic tobacco. PLOS ONE. 10 (10), e0139392. doi: 10.1371/journal.pone.0139392

Zhu, H., Zhang, T.-J., Zheng, J., Huang, X.-D., Yu, Z.-C., Peng, C.-L., et al. (2018). Anthocyanins function as a light attenuator to compensate for insufficient photoprotection mediated by nonphotochemical quenching in young leaves of Acmena acuminatissima in winter. Photosynthetica 56, 445-454. doi: 10.1007/ s11099-017-0740-1

Conflict of Interest: The authors declare that the research was conducted in the absence of any commercial or financial relationships that could be construed as a potential conflict of interest.

Copyright (C) 2020 Gong, Liu, Wang, Chen, Martin and Meng. This is an open-access article distributed under the terms of the Creative Commons Attribution License (CC BY). The use, distribution or reproduction in other forums is permitted, provided the original author(s) and the copyright owner(s) are credited and that the original publication in this journal is cited, in accordance with accepted academic practice. No use, distribution or reproduction is permitted which does not comply with these terms. 